\title{
The Serum Citrulline and D-Lactate are Associated with Gastrointestinal Dysfunction and Failure in Critically III Patients
}

\author{
Jin Teng' \\ Lu Xiang ${ }^{2}$ \\ Huaicong Long ${ }^{2}$ \\ Caiping $\mathrm{Gao}^{3}$ \\ Lei Lei $^{3}$ \\ Yinghui Zhang ${ }^{3}$ \\ 'Department of Cadre Health, Sichuan \\ Academy of Medical Sciences \& Sichuan \\ Provincial People's Hospital, Chengdu \\ City, Sichuan Province, 610072, People's \\ Republic of China; ${ }^{2}$ Department of \\ Geriatrics, Sichuan Academy of Medical \\ Sciences \& Sichuan Provincial People's \\ Hospital, Chengdu City, Sichuan \\ Province, 610072, People's Republic of \\ China; ${ }^{3}$ Department of Digestive Internal \\ Medicine, Sichuan Academy of Medical \\ Sciences \& Sichuan Provincial People's \\ Hospital, Chengdu City, Sichuan \\ Province, 610072, People's Republic of \\ China
}

Objective: This study attempted to screen and combine effective biomarkers to analyse the association between these biomarkers and gastrointestinal failure (GIF) in critically ill patients. Methods: A total of 110 critically ill patients with acute gastrointestinal injury (AGI) admitted to ICU were enrolled. The AGI grade was determined by the AGI classification proposed by ESICM. There were 67 patients in gastrointestinal dysfunction (GID) group (AGI grade II), 43 patients in GIF group (AGI grade III-IV), and 41 healthy adults in healthy control (HC) group. APACHE II and SOFA score were used to evaluate the disease severity. Peripheral blood samples of patients were collected within 24 hours of admission to the ICU (prior-treatment) and after the conventional medication therapy for 7 consecutive days (post-treatment). Citrulline serum level was detected by high-performance liquid chromatography-tandem mass spectrometry (HPLCMS/MS) method, and D-lactate and lipopolysaccharide (LPS) serum levels were measured by ELISA. Pearson correlation, logistic regression, and ROC curve analysis were used.

Results: Patients with GID or GIF had lower serum level of citrulline, while higher levels of D-lactate and LPS than HC. Compared with GID patients, serum level of citrulline was reduced, while D-lactate and LPS were elevated in GIF patients. Correlation analysis displayed that serum levels of citrulline, D-lactate, and LPS were associated with the APACHE II and SOFA score in patients with GID or GIF. Logistics regression analysis showed that citrulline and D-lactate were risk for both GID and GIF. ROC curve analysis exhibited that combination of citrulline and D-lactate had relatively high value to distinguish GID from HC, GIF from GID, and GIF from HC.

Conclusion: Serum citrulline and D-lactate were associated with severity of GIF, combination of citrulline and D-lactate improved the diagnostic efficacy to identify GIF in critically ill patients.

Keywords: gastrointestinal failure, gastrointestinal dysfunction, citrulline, D-lactate, critically ill patients

\section{Introduction}

Gastrointestinal failure (GIF), defined as concomitant occurrence of $\geq$ three gastrointestinal symptoms, is common in ICU patients. ${ }^{1}$ Among critically ill patients, the precipitating factors of GIF include inflammation, sepsis, electrolyte abnormalities, and acidosis. ${ }^{2}$ At present, there is no single biomarker that could reliably describe GIF. ${ }^{3}$ GIF is a clinical entity that often goes undiagnosed. ${ }^{4}$ Patients with GIF have higher mortality rate than patients without GIF. ${ }^{5}$ Therefore, correct diagnosis and early start of treatment are essential for GIF.
Correspondence: Yinghui Zhang Department of Digestive Internal Medicine, Sichuan Academy of Medical Sciences \& Sichuan Provincial People's Hospital, Chengdu City, Sichuan Province, 610072, People's Republic of China Tel +86- 18981838I23

Email jn2021680@I63.com 
In humans, the main source of citrulline is synthesized by enterocytes, and the level of circulating citrulline is regarded as a biomarker indicative of a functional intestine ${ }^{6,7}$ Citrulline is a biomarker of intestinal failure due to enterocyte mass decrease. ${ }^{8}$ Plasma citrulline serves as an indicator of small bowel functional mass, which is used in clinical practice. ${ }^{9}$ An animal study has exhibited that serum citrulline acts as a biomarker to diagnose septic acute intestinal dysfunction in rat. ${ }^{10}$ Notably, both gastrointestinal dysfunction (GID) and GIF are related to low level of citrulline in critically ill patients. ${ }^{11}$ Therefore, the diagnostic role of serum citrulline on GIF need to be further investigated.

D-lactate is produced in minimal quantities by mammal cells, ${ }^{12}$ D-lactate level can elevate because of excess gastrointestinal microbial production. ${ }^{13}$ D-lactate has been used for the diagnosis of gastrointestinal diseases. For instances, plasma D-lactate level markedly raised early in necrotizing enterocolitis (NEC), and is correlated with extensive disease in NEC infants. ${ }^{14}$ Serum D-lactate is regarded as a valuable diagnostic marker for appendicitis. ${ }^{15}$ D-lactate is a useful diagnostic marker for intestinal ischemia induced by abdominal compartment syndrome. ${ }^{16}$ Serum D-lactate improves the diagnosis efficacy of intestinal ischemia in acute abdomen patients. ${ }^{17}$ However, the diagnostic value of serum citrulline combined with serum D-lactate to identify GIF is still unknown.

In this study, we explored the association between the serum biomarkers (citrulline, D-lactate, and LPS) and GID/GIF in critically ill patients, and further evaluated the diagnostic efficacy of citrulline and D-lactate for GID/GIF. Our research may provide the potential serum biomarkers to aid in the diagnosis of GID or GIF.

\section{Materials and Methods}

\section{Patients}

A total of 506 patients who hospitalized in the ICU of our hospital (including the department of the surgical ICU, geriatric ICU, and neurosurgical ICU) from March 2018 to March 2019 were retrospectively analyzed. Inclusion criteria: (i) Patients more than 18 years old; (ii) Patients who stayed in the ICU for more than 24 hours; (iii) Patients with acute gastrointestinal injury (AGI) grade II-IV. Exclusion criteria: (i) Patients who stayed in the ICU for less than 24 hours; (ii) Patients with acute kidney injury and chronic kidney disease (citrulline is metabolized by the renal, and renal dysfunction can affect citrulline level); (iii) Patients with chronic gastrointestinal disease or previous history of intestinal resection; (iv) Patients without AGI (AGI $=0)$ and patients with AGI grade I. A total of 110 patients were screened out and included in this study. The AGI grade was assessed daily according to recommendation of the AGI classification proposed by European Society of Intensive Care Medicine (ESICM, 2012) ${ }^{18}$ (AGI grade I, risk of GID or GIF; AGI grade II, GID; AGI grade III, GIF; AGI grade IV, GIF with severe impact on distant organ function) during the first week of the subject's ICU stay. The determination of AGI grade was based on the mean value of AGI grade. According to the AGI grade, 110 patients were divided into GID group (AGI grade II, $\mathrm{n}=$ 67) and GIF group (AGI grade III-IV, $n=43$ ). Acute Physiology and Chronic Health Evaluation (APACHE) II score $^{19}$ and Sepsis-related Organ Failure Assessment (SOFA) score ${ }^{20}$ were used to evaluate the disease severity of patients. During the same period, 41 healthy adults who underwent physical examination in the outpatient department of our hospital were selected as healthy control (HC). This study was approved by the Medical Ethics Committee of our Hospital, and written informed consent was obtained from all close relatives of patients. This study was conducted in accordance with the Declaration of Helsinki.

\section{Preparation and Storage of Serum}

Peripheral blood samples of patients were collected within 24 hours of admission to the ICU (Prior-treatment) and after the conventional medication therapy for 7 consecutive days (Post-treatment). Peripheral blood samples of $\mathrm{HC}$ were collected during the normal physical examination. Peripheral blood samples of both patients and $\mathrm{HC}$ were centrifuged at $3000 \mathrm{rpm}$ at $4^{\circ} \mathrm{C}$ for $10 \mathrm{~min}$. The serum was collected and frozen at $-20^{\circ} \mathrm{C}$ for subsequent experiments. Notably, serum samples were frozen for no more than one month before testing.

\section{Laboratory Measurements}

Protein precipitation of serum samples was performed in 96 well Strata Impact $2 \mathrm{~mL}$ filtration plates. To each well was added $490 \mu \mathrm{L}$ acetonitrile: water: formic acid (85: 14.8: 0.2 $\mathrm{v} / \mathrm{v}$ ) containing internal standard. This was followed by the addition of $10 \mathrm{~mL}$ of serum. After mixing gently, the plate was covered, allowed to stand for $5 \mathrm{~min}$, and the filtrate was collected under vacuum. The 96-well collection plate was loaded into the Acquity sample manager and the sample was injected onto the analytical column. Then, the serum level of citrulline was detected by the method of highperformance liquid chromatography-tandem mass spectrometry (HPLC-MS/MS) on an Agilent 1200 HPLC system 
(Agilent, Santa Clara, CA, USA). Solvent A and $B$ consisted of $10 \mathrm{mM}$ ammonium formate with $0.1 \%$ formic acid in water and acetonitrile with $0.1 \%$ formic acid, respectively. The gradient program was $0.0-0.5 \mathrm{~min}, 70 \%$ $\mathrm{B}$; 0.5-1.0 min, gradient to $10 \% \mathrm{~B} ; 1.0-3.5 \mathrm{~min}, 10 \% \mathrm{~B}$; 3.5-4.0 min, gradient to $70 \% \mathrm{~B}$; and $4.0-6.0 \mathrm{~min}, 70 \%$ B. The flow rate was to $0.6 \mathrm{~mL} / \mathrm{min}$ during all separation steps and injection volume was $10 \mu \mathrm{L}$. Detection was conducted in the positive-ion mode and the electrospray ionization source parameters were as follows: spray voltage, 3000; capillary temperature, 325; sheath gas pressure, 60; ion sweep gas pressure, 0.2 ; capillary offset, 10; and tube lens offset, 50-75. In addition, the serum level of D-lactate was measured by human D-lactate ELISA kit (SBJ-H1970; Nanjing SenBeiJia Biological Technology Co., Ltd; Nanjing; http://www.sbjbio.com/Products/D4865.html). The serum level of lipopolysaccharide (LPS) was detected by human LPS ELISA kit (ml061109; mlbio; Shanghai; https://www.mlbio.cn/article-20.html). The reference value is 8.49 to $59.43 \mu \mathrm{mol} / \mathrm{L}$ for serum citrulline, 1.17 to $3.45 \mathrm{pg} /$ $\mathrm{mL}$ for serum LPS, and 3.23 to $10.37 \mu \mathrm{mol} / \mathrm{L}$ for serum D-Lactate, respectively.

\section{Statistical Analysis}

Data were statistically analyzed using SPSS 23.0 (SPSS Inc., Chicago, IL, USA). Continuous data were presented as means \pm standard deviations (SD) and evaluated by a Student's $t$-test when the data were normally distributed. Enumeration data were presented as numbers $(\mathrm{N})$ and assessed using a $\chi^{2}$ test. The measurement of serum biomarker levels was performed in triplicates, and was repeated three times. Correlation significance was evaluated by Pearson correlation analysis. Correlation analysis of serum biomarkers and GID/GIF was performed using a logistic regression model. The receiver operating characteristic (ROC) curve was plotted to assess the validity of the potential biomarkers to diagnose GID/GIF. For the analysis of combined variable, logistic regression analysis (whether the patients were GID/GIF or not GID/GIF served as the dependent variable; citrulline and D-lactate served as the covariate) was conducted to determine the prediction probability value. The ROC curve was plotted by using the prediction probability value. The 95\% confidence intervals (CI) and area under the ROC curve (AUC) were defined. According to the data of the $95 \% \mathrm{CI}$ and AUC, the cut-off value, sensitivity, and specificity were obtained. A P value $<0.05$ was considered statistically significant.

\section{Results}

\section{Comparison of Clinical Data Between Health Control and Patients}

As exhibited in Tables 1, 67 patients were in GID group (AGI grade I-II), including 33 males and 34 females, with an average age of $(51.36 \pm 17.50)$ years; There were 43 patients in GIF group (AGI grade III-IV), including 25 males and 18 females, with an average age of $(49.07 \pm 23.66)$ years. The HC group included 18 males and 23 females, with an average age of $(48.56 \pm 16.31)$ years old. There was no significant

Table I Baseline Characteristics of Patients and Healthy Control (HC)

\begin{tabular}{|c|c|c|c|c|}
\hline Characteristic & $\mathrm{HCN}=41$ & $\begin{array}{l}\text { Gastrointestinal Dysfunction (GID) } \\
N=67\end{array}$ & $\begin{array}{l}\text { Gastrointestinal Failure (GIF) } \\
N=43\end{array}$ & $P$ value \\
\hline Age $(y)$ & $48.56 \pm|6.3|$ & $51.36 \pm 17.50$ & $49.07 \pm 23.66$ & \\
\hline$<50$ & 22 & 36 & 27 & 0.3159 \\
\hline$\geq 50$ & 19 & 31 & 16 & \\
\hline \multicolumn{5}{|l|}{ Gender (cases) } \\
\hline Male & 18 & 33 & 25 & 0.2304 \\
\hline Female & 23 & 34 & 18 & \\
\hline APACHE-II score & - & $12.34 \pm 2.26$ & $18.77 \pm 3.60$ & $<0.001 \#$ \\
\hline SOFA score & - & $3.92 \pm 1.49$ & $7.86 \pm 1.85$ & $<0.001 \#$ \\
\hline LPS (pg/mL) & $2.35 \pm 0.72$ & $5.27 \pm 0.99$ & $8.91 \pm 2.97$ & $<0.001 * \#$ \\
\hline D-Lactate $(\mu \mathrm{mol} / \mathrm{L})$ & $8.17 \pm 2.14$ & $16.29 \pm 5.33$ & $22.26 \pm 9.1$ & $<0.001 * \#$ \\
\hline Citrulline ( $\mu \mathrm{mol} / \mathrm{L})$ & $|5.66 \pm 2.8|$ & $13.13 \pm 1.96$ & $10.04 \pm 2.36$ & $<0.001 * \#$ \\
\hline
\end{tabular}

Notes: $* \mathrm{P}<0.001$ vs HC, \#P $<0.001$ vs GID.

Abbreviation: LPS, lipopolysaccharide. 
difference in age and gender between HC and GID group, between HC and GIF group, or between GID and GIF group (all $\mathrm{P}>0.05$ ). A significant increase was found in the APACHE-II and SOFA score in the GIF group compared with those in the GID group $(\mathrm{P}<0.001)$. Compared with the HC group, the serum levels of D-lactate and LPS were significantly increased, while the serum level of citrulline was significantly decreased in the GID and GIF group $(\mathrm{P}<$ 0.001). Compared with the GID group, the serum levels of D-lactate and LPS were markedly elevated, while serum level of citrulline was markedly reduced in GIF group $(\mathrm{P}<0.001)$.

\section{Correlation Analysis Between the Serum Levels of Citrulline, D-Lactate, and LPS and the APACHE-II and SOFA Score}

APACHE-II and SOFA score were used to evaluate the disease severity of critically ill patients. As shown in Figure $1 \mathrm{~A}$ and $\mathrm{B}$, the serum level of citrulline was negatively correlated with APACHE-II score $(\mathrm{r}=-0.4476, \mathrm{P}<0.001)$ and SOFA score $(\mathrm{r}=-0.4194, \mathrm{P}<0.001)$ in critically ill patients. Additionally, there was a positive correlation between the serum level of D-lactate and APACHE-II score $(\mathrm{r}=0.4853, \mathrm{P}<0.001)$ or SOFA score $(\mathrm{r}=0.510, \mathrm{P}<0.001)$ in critically ill patients (Figure $1 \mathrm{C}$ and D). Moreover, a positive correlation between the serum level of LPS and APACHE-II score $(\mathrm{r}=0.7612, \mathrm{P}<0.001)$ or SOFA score $(\mathrm{r}=$ $0.6132, \mathrm{P}<0.001)$ was observed in critically ill patients (Figure 1E and F).

\section{Citrulline Serum Level Was Elevated While D-Lactate and LPS Serum Levels Were Reduced in Critically III Patients After Treatment}

Paired analysis was performed on serum samples of critically ill patients within $24 \mathrm{~h}$ of ICU admission (Priortreatment) and after the conventional medication therapy for 7 consecutive days (Post-treatment). After treatment, 52 out of 67 patients in the GID group and 33 out of 43 patients in the GIF group displayed an increase in citrulline serum level (all $\mathrm{P}<0.001$, Figure $2 \mathrm{~A}$ ). As shown in Figure $2 \mathrm{~B}$ and $\mathrm{C}$, the serum level of D-lactate was decreased in 48 of the 67 GID patients, and LPS was decreased in 43 of the 67 GID patients after treatment (all $\mathrm{P}<0.05$ ). The serum level of D-lactate was declined in 34 of the 43 GIF patients, and LPS was declined in 27 of the 43 GIF patients after treatment (all $\mathrm{P}<0.05$ ).

\section{Logistic Regression Analysis of Related Serum Biomarkers for GID/GIF}

Logistic regression analysis was conducted to evaluate the associations between serum biomarkers and GID/GIF. As shown in Table 2, there was a significant correlation between GID and the serum citrulline and D-lactate $(\mathrm{P}<0.001)$, but there was no correlation between GID and serum LPS (P $>$ 0.05). As displayed in Table 3, the GIF was correlated with the serum citrulline and D-lactate $(\mathrm{P}<0.01)$, while had no correlation to the serum LPS $(\mathrm{P}>0.05)$.

\section{Diagnostic Value of Serum Citrulline and D-Lactate in GID and GIF}

ROC curve analysis was used for assessing the diagnostic values of citrulline and D-lactate (Figure 3A-F and Table 4). Serum citrulline cannot distinguish GID from HC (AUC = 0.7472 , sensitivity $=67.16 \%$, specificity $=70.73 \%$ ) or GIF from GID (AUC $=0.8112$, sensitivity $=67.44 \%$, specificity $=$ $80.06 \%$ ). Serum citrulline can distinguish GIF from $\mathrm{HC}$ to some extent $(\mathrm{AUC}=0.9314$, sensitivity $=74.42 \%$, specificity $=87.08 \%$ ). In addition, serum D-lactate can distinguish GID from $\mathrm{HC}(\mathrm{AUC}=0.9337$, sensitivity $=83.58 \%$, specificity $=$ $87.80 \%)$ or GIF from $\mathrm{HC}(\mathrm{AUC}=0.9512$, sensitivity $=$ $86.05 \%$, specificity $=87.80 \%$ ) to some extent. Serum D-lactate cannot distinguish GIF from GID (AUC $=0.8770$, sensitivity $=72.09 \%$, specificity $=88.06 \%$ ).

\section{Diagnostic Value of the Combination of Citrulline and D-Lactate in the Diagnosis of GID and GIF}

To further improve the diagnostic value, the combination of serum citrulline and D-lactate was analyzed by ROC curve (Figure 4A-C and Table 5). The combination of serum citrulline and D-lactate can distinguish GID from $\mathrm{HC}$ $(\mathrm{AUC}=0.9698$, sensitivity $=91.04 \%$, specificity $=97.56 \%)$, GIF from GID (AUC $=0.9210$, sensitivity $=81.40 \%$, specificity $=89.71 \%$ ), and GIF from $\mathrm{HC}(\mathrm{AUC}=0.9943$, sensitivity $=95.35 \%$, specificity $=97.56 \%$ ).

\section{Discussion}

Evaluation of GIF is still limited today to clinical and radiological assessment, the promising serum biomarkers needs to be investigated. ${ }^{3}$ It has been documented that several serum biomarkers such as citrulline, ${ }^{9}$ D-lactate, ${ }^{16}$ 
A

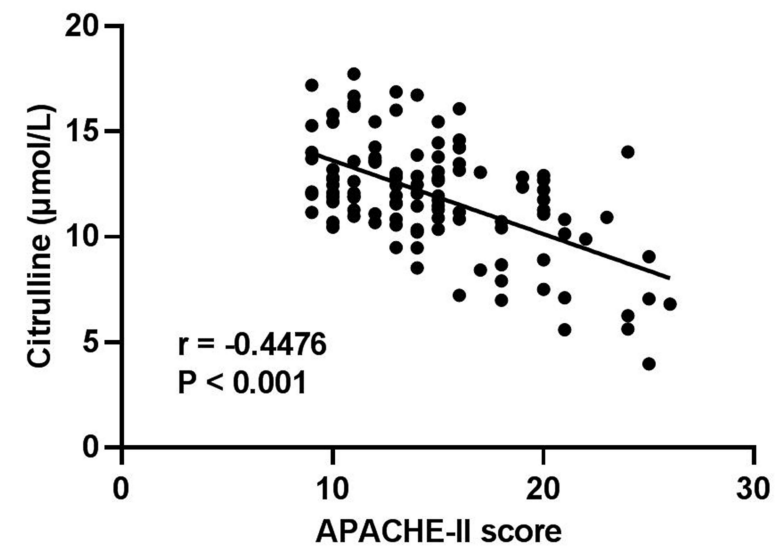

C

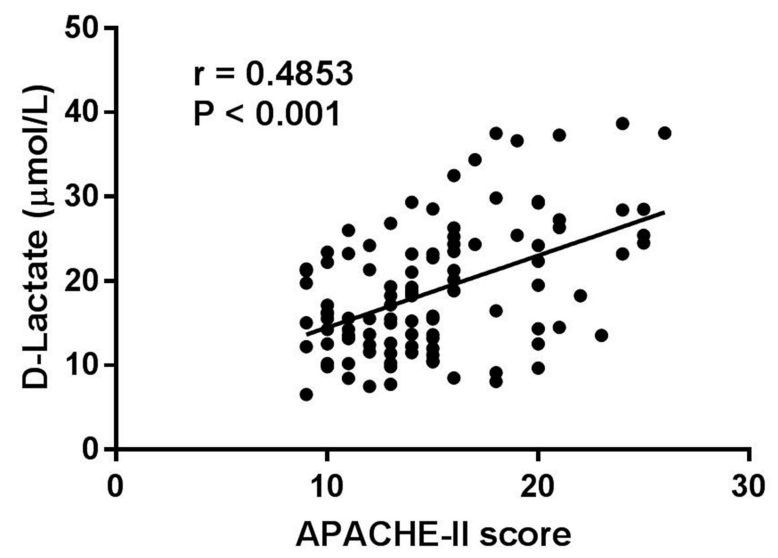

E

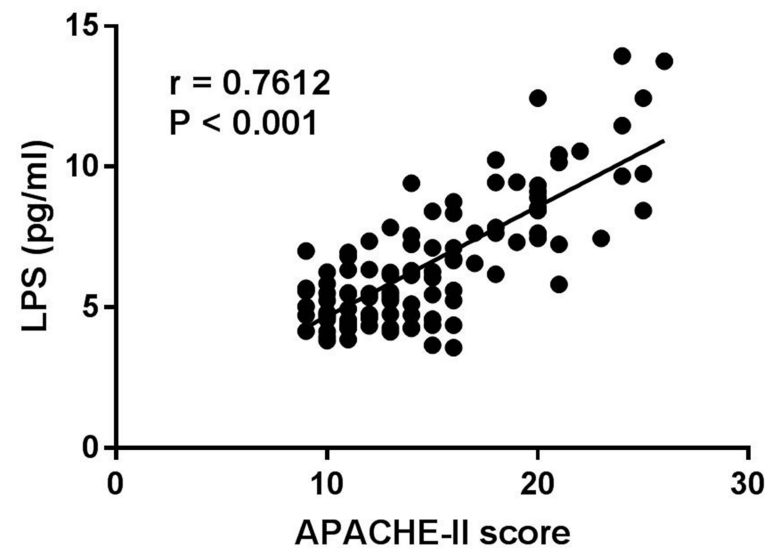

B

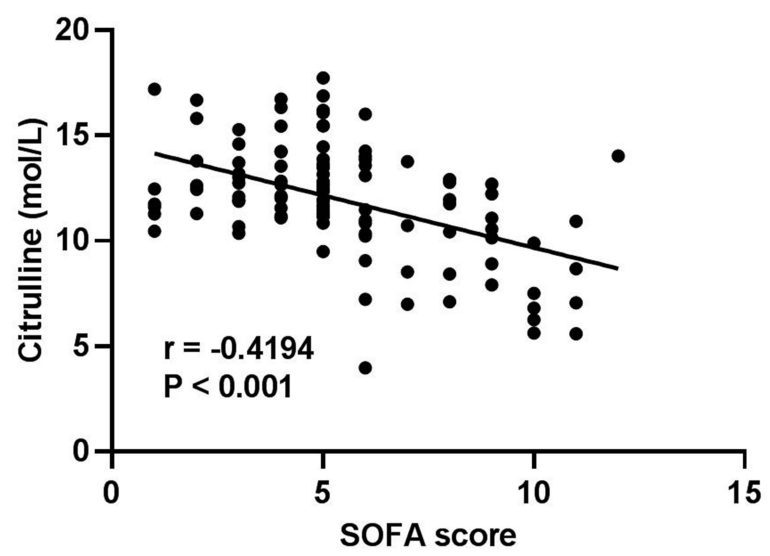

D

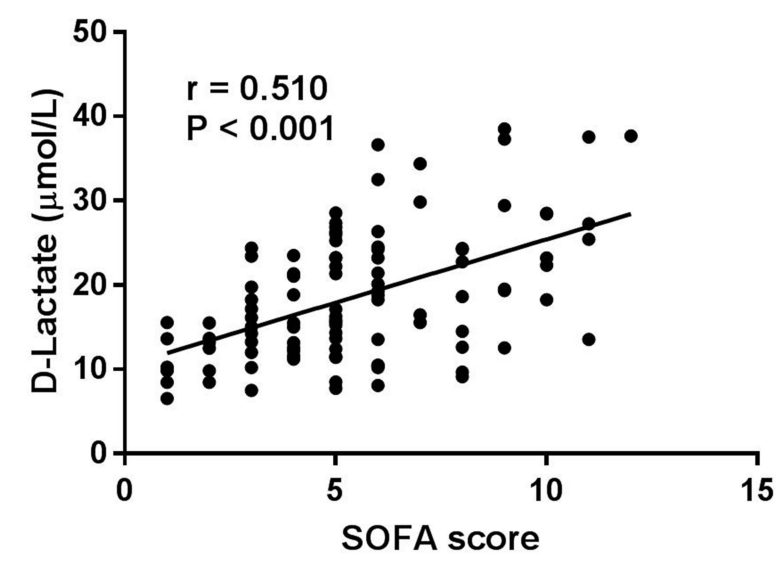

F

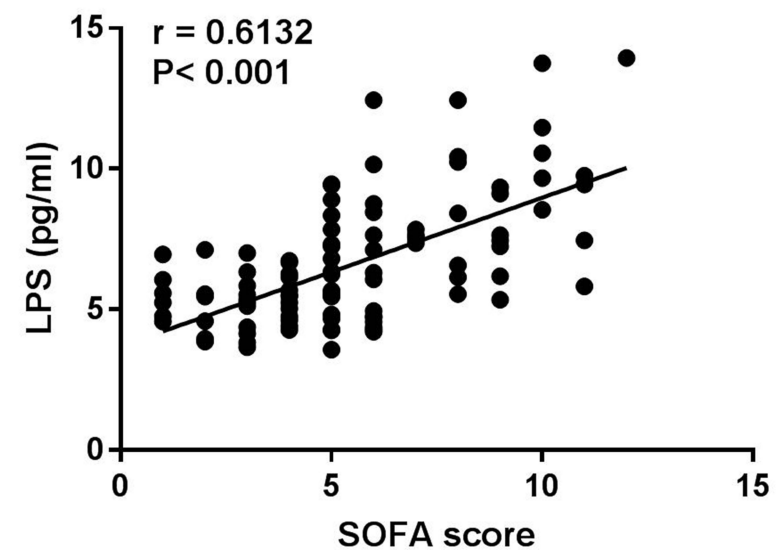

Figure I Correlation analysis between the serum levels of citrulline, D-lactate, and lipopolysaccharide (LPS) and the APACHE-II and SOFA scores. (A) Citrulline was negatively correlated with APACHE-II score; (B) citrulline was negatively correlated with SOFA score; (C) D-lactate was positively correlated with APACHE-II score; (D) D-lactate was positively correlated with SOFA score; (E) LPS was positively correlated with APACHE-II score; (F) LPS was positively correlated with SOFA score.

and LPS ${ }^{21}$ act as diagnostic markers for different gastrointestinal diseases. Here, patients with GID or GIF had significantly lower serum level of citrulline, while higher levels of D-lactate and LPS than HC. Compared with GID patients, the serum level of citrulline was markedly reduced, while D-lactate and LPS were markedly elevated 


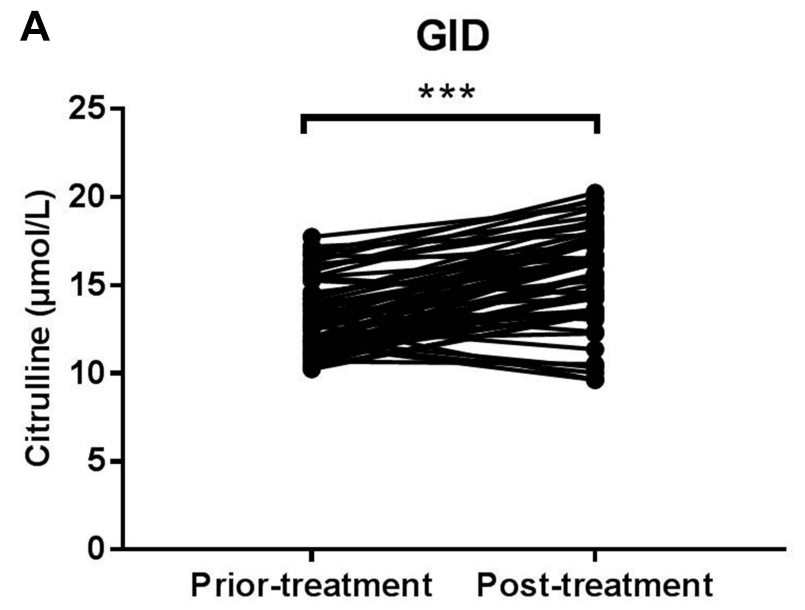

B

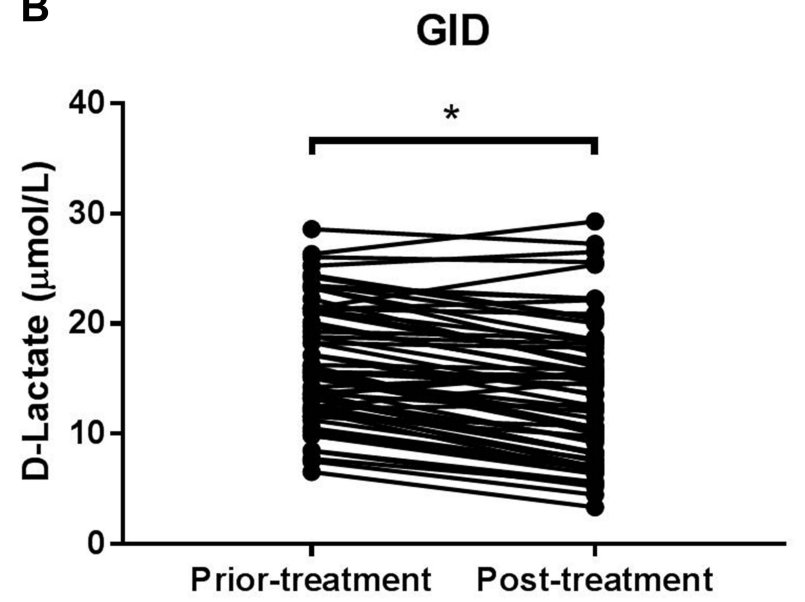

C

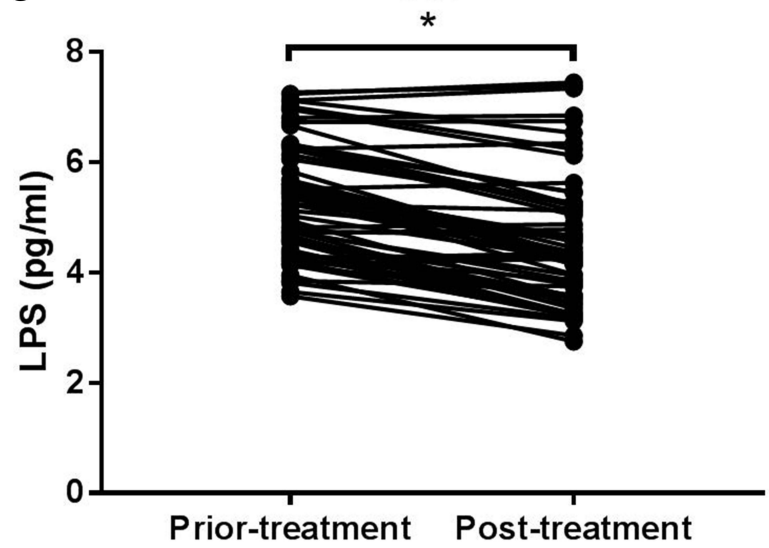

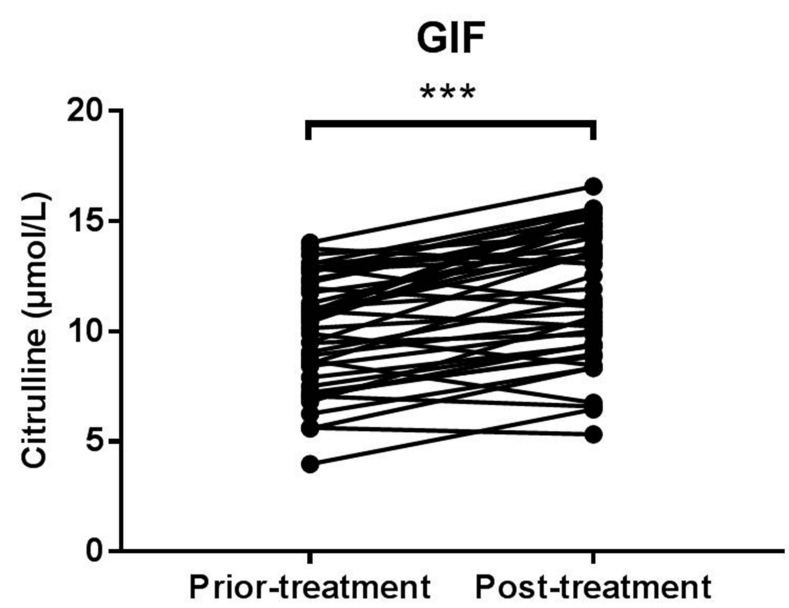
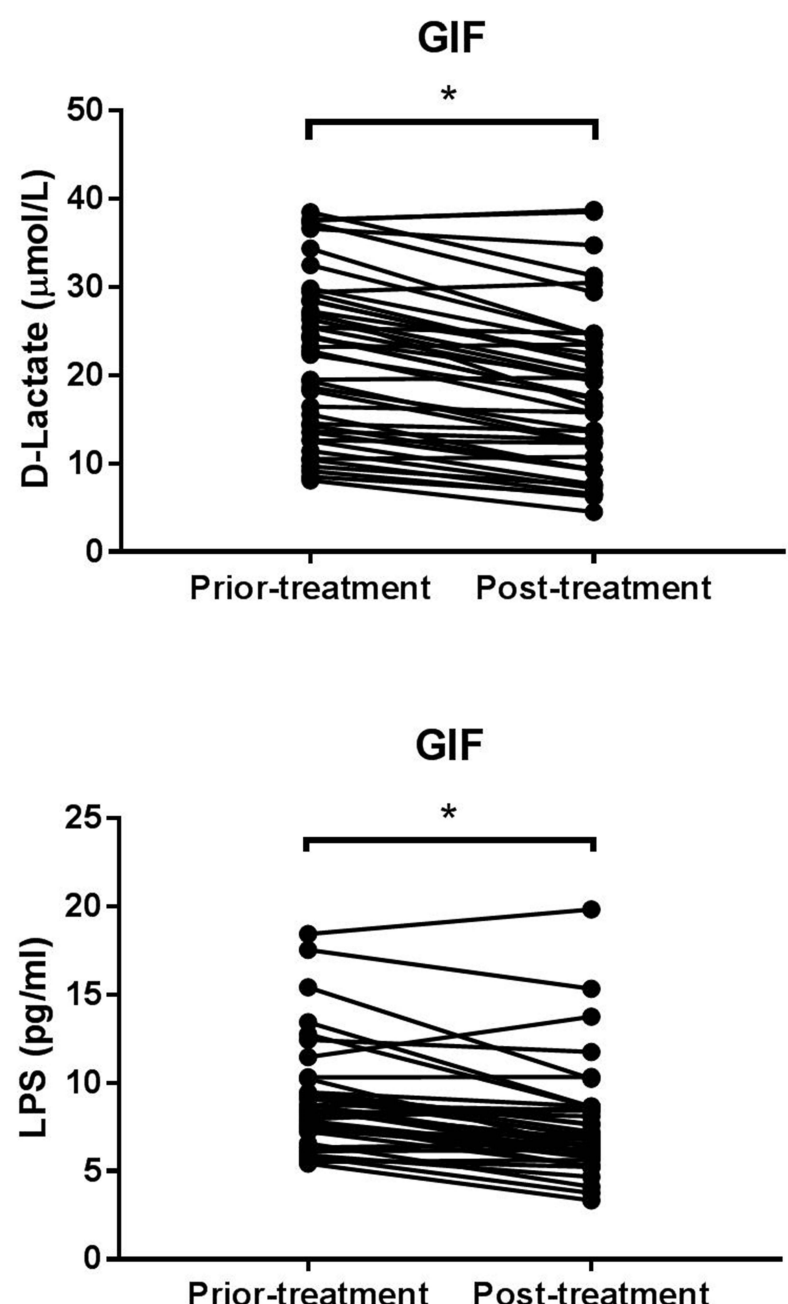

Figure 2 Citrulline serum level was elevated while D-lactate and lipopolysaccharide (LPS) serum levels were reduced in critically ill patients after treatment. (A) There were 52 out of 67 patients in the gastrointestinal dysfunction (GID) group and 33 out of 43 patients in the gastrointestinal failure (GIF) group displayed an increase in citrulline serum level after treatment. ***P $<0.00$ I vs Prior-treatment; (B) there were 48 out of 67 patients in the GID group and 34 out of 43 patients in the GIF group exhibited a decrease in D-lactate serum level after treatment. *P $<0.05$ vs Prior-treatment; (C) there were 43 out of 67 patients in the GID group and 27 out of 43 patients in the GIF group showed an increase in LPS serum level after treatment. $* \mathrm{P}<0.05$ vs Prior-treatment. 
Table 2 Logistic Regression Analysis Was Performed to Analyze the Association Between the Serum Biomarkers (Citrulline, D-Lactate, and LPS) and Gastrointestinal Dysfunction (GID)

\begin{tabular}{|l|l|l|l|l|}
\hline Projects & B & Sig. & Exp (B) & $\mathbf{9 5 \%}$ Cl for Exp (B) \\
\hline Citrulline $(\mu \mathrm{mol} / \mathrm{L})$ & -0.399 & 0.000 & 0.671 & $0.558-0.807$ \\
D-Lactate $(\mu \mathrm{mol} / \mathrm{L})$ & 0.751 & 0.000 & 2.120 & $1.541-2.916$ \\
\hline
\end{tabular}

Table 3 Logistic Regression Analysis Was Performed to Analyze the Association Between the Serum Biomarkers (Citrulline, D-Lactate, and LPS) and Gastrointestinal Failure (GIF)

\begin{tabular}{|l|l|l|l|l|}
\hline Projects & B & Sig. & Exp (B) & $\mathbf{9 5 \% ~ C l ~ f o r ~ E x p ~ ( B ) ~}$ \\
\hline Citrulline $(\mu \mathrm{mol} / \mathrm{L})$ & -0.963 & 0.000 & 0.382 & $0.242-0.604$ \\
D-Lactate $(\mu \mathrm{mol} / \mathrm{L})$ & 0.821 & 0.001 & 2.272 & $1.413-3.653$ \\
\hline
\end{tabular}

in GIF patients, suggesting that serum citrulline, D-lactate, and LPS may be related to GID or GIF. Additionally, a significant increase was found in the APACHE-II and SOFA score in GIF patients compared with GID patients. Clinically, APACHE-II and SOFA score are often used to distinguish between different AGI grades in critically ill patients. $^{22}$ The critically ill patients with secondary AGI have a higher APACHE-II score. ${ }^{23}$ Increased SOFA score is associated with the development of AGI II in critically ill patients. ${ }^{24}$ We assume that the APACHE-II and SOFA score may reflect the disease severity of GID or GIF. To further explore the relevance between these biomarkers and the disease severity, we assessed the correlation between these biomarkers and the APACHE-II and
SOFA score in GID or GIF patients. In this study, Pearson's correlation analysis displayed that citrulline serum level was negatively correlated with APACHE-II and SOFA score, while D-lactate and LPS serum levels were positively correlated with APACHE-II and SOFA score in critically ill patients. Taken together, our data suggested that the serum levels of citrulline, D-lactate, and LPS may be correlated with the severity of GID in critically ill patients.

Citrulline plasma level serves as a marker for enterocyte metabolic mass, the level often decreases during intestinal dysfunction. ${ }^{25}$ In the present study, approximately $77.61 \%$ of GID patients and $76.74 \%$ of GIF patients displayed an increase in citrulline serum level following treatment. Accumulation of D-lactate is induced by dysfunction of metabolic systems and abnormal overgrowth of D-lactate-producing bacteria in the intestine. ${ }^{26}$ In this study, the D-lactate serum level was decreased in about $71.64 \%$ of GID patients and $79.07 \%$ of GIF patients after treatment. LPS is a prototypic microbe-derived inflammatory signal, the intestinal bacteria is regarded as the major source of LPS. ${ }^{27}$ Here, approximately $64.18 \%$ of GID patients and $79.41 \%$ of GIF patients exhibited a decrease in LPS serum level after treatment. Taken together, our results indicated that monitoring changes in these biomarkers following treatment may reflect the AGI grade to some extent in critically ill patients.

Previous studies have determined that citrulline levels are correlated with disease severity in enteropathies. ${ }^{28,29}$
A
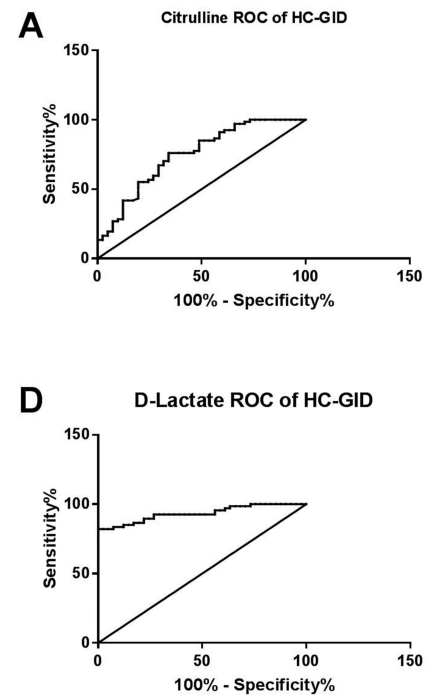
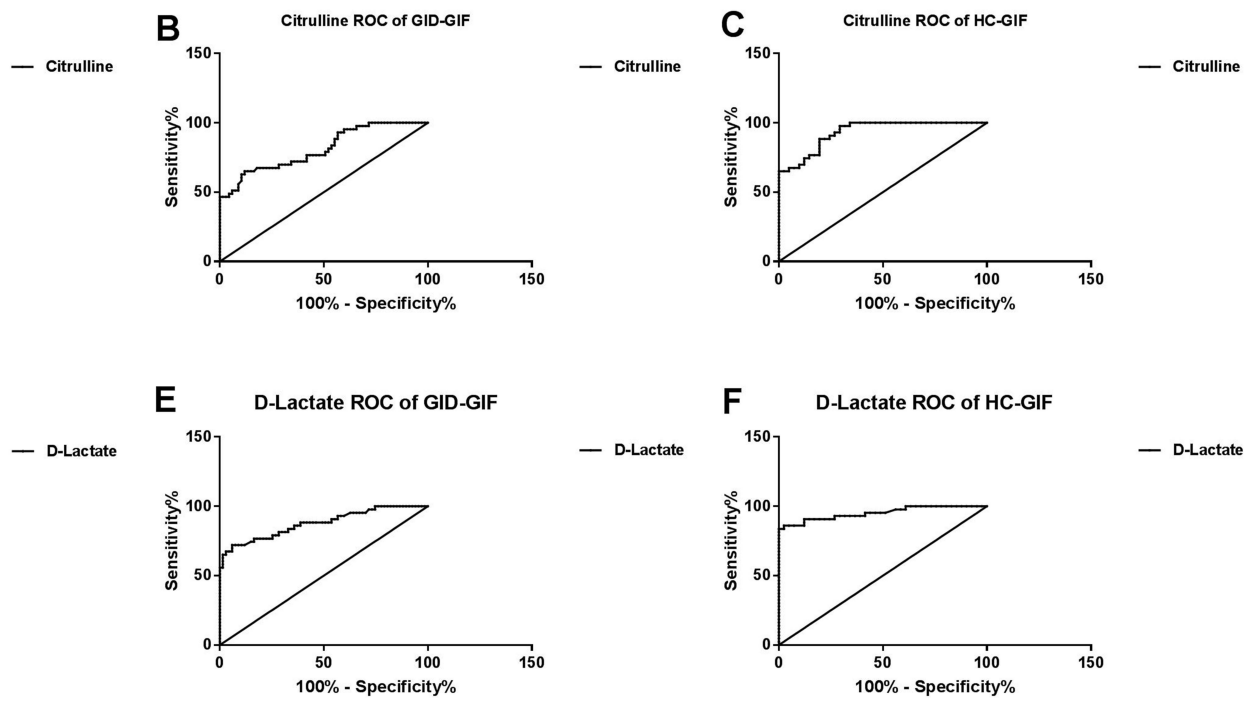

E

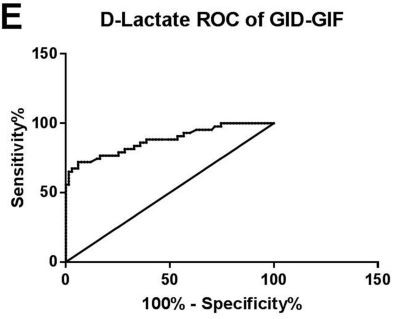

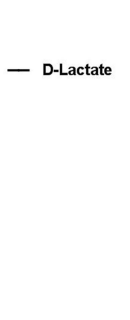

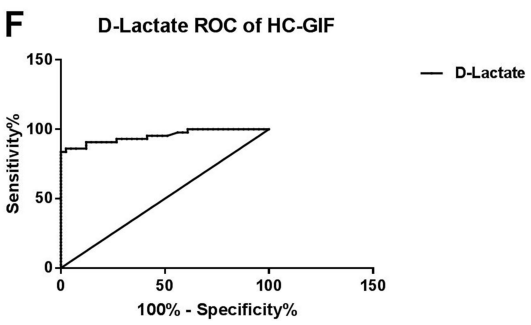

Figure 3 Diagnostic value of serum citrulline or D-lactate in gastrointestinal failure (GID) and gastrointestinal failure (GIF). (A-C) ROC curve analysis was used to assess the value of serum citrulline to distinguish GID from healthy control (HC), GIF from GID, or GIF from HC; (D-F) ROC curve analysis was used for evaluating the value of serum D-lactate to distinguish GID from HC, GIF from GID, or GIF from HC. 
Table 4 Diagnostic Accuracies of Serum Biomarkers of Gastrointestinal Dysfunction (GID) and Gastrointestinal Failure (GIF)

\begin{tabular}{|l|l|l|l|l|l|l|l|}
\hline & Groups & Cutoff & Sensitivity (\%) & Specificity (\%) & AUC & 95\% CI & P value \\
\hline \multirow{5}{*}{ Citrulline } & HC-GID & 13.80 & 67.16 & 70.73 & 0.7472 & $0.6506-0.8438$ & $<0.000$ I \\
& GID-GIF & 11.38 & 67.44 & 80.60 & $0.8 I I 2$ & $0.693-0.9319$ & $<0.000$ I \\
& HC-GIF & 12.27 & 74.42 & 87.08 & $0.93 I 4$ & $0.8828-0.9800$ & $<0.0001$ \\
\hline \multirow{5}{*}{ D-Lactate } & HC-GID & 10.54 & 83.58 & 87.8 & 0.9337 & $0.8344-0.9753$ & $<0.0001$ \\
& GID-GIF & 23.25 & 72.09 & 88.06 & 0.8770 & $0.8071-0.9468$ & $<0.0001$ \\
& HC-GIF & 10.56 & 86.05 & 87.80 & $0.95 I 2$ & $0.9064-0.996 I$ & $<0.0001$ \\
\hline
\end{tabular}

Abbreviations: HC, healthy control; AUC, area under the ROC curve.

Notably, citrulline acts as a biomarker for GID in the critically ill. $^{30}$ In this study, we found that the serum citrulline was associated with the GID or GIF in critically ill patients, suggesting that serum citrulline may serve as an important biomarker for GID or GIF in auxiliary diagnosis. It has been documented that the level of D-lactate often increases in gastrointestinal disorders such as intestinal barrier dysfunction ${ }^{31}$ and necrotizing enterocolitis. ${ }^{14}$ Importantly, elevated D-lactate level increases the odds of AGI grade II in critically ill patients. ${ }^{22}$ Here, the serum citrulline was related to the GID or GIF in critically ill patients, indicating that serum D-lactate may be associated with the diagnosis of GID or GIF. To further assess the valid of serum citrulline and D-lactate in the diagnosis of GID or GIF, ROC analysis was performed. Our results revealed that single biomarkers (citrulline or D-lactate) were not effective in the diagnosis of GID or GIF.

Existing document has demonstrated that the diagnostic value of the combination biomarkers was higher than any single biomarkers in the detection of intestinal barrier dysfunction. ${ }^{31}$ To further improve the diagnostic efficacy, the combination of serum citrulline and D-lactate was analyzed by ROC curve. Previous studies have displayed that the serum levels of citrulline is reduced while D-lactate is raised in different gastrointestinal diseases. ${ }^{32,33}$ Notably, the combination of serum citrulline,
D-lactate, and I-FABP can more effectively diagnose intestinal barrier dysfunction than any single biomarker in patients after major abdominal surgery. ${ }^{31}$ Here, our results revealed that the AUC, sensitivity, and specificity of the combination of serum citrulline and D-lactate were high, which can distinguish GID from HC, GIF from GID, and GIF from HC. To sum up, the combination of serum citrulline and D-lactate may improve the diagnostic efficacy of a single biomarker in GIF.

\section{Limitations}

There were several limitations to our study. First, the sample size was relatively small, and it's difficult to exclude the effects of confounding factors because of the diverse baseline characteristics of patients. Second, the set of biomarkers may be expensive and labour-intensive in clinical practice and more simplified diagnostic strategies still need to be studied in the future. Third, there were fewer time points to assess the disease severity (APACHE II and SOFA score) and the serum levels of biomarkers. Fourth, more indicators should be included for the assessment of GID/GIF rather than only adopting the subjective assessment of GID/GIF.

\section{Conclusions}

In summary, we found that the serum citrulline and D-lactate were associated with the severity of GID in
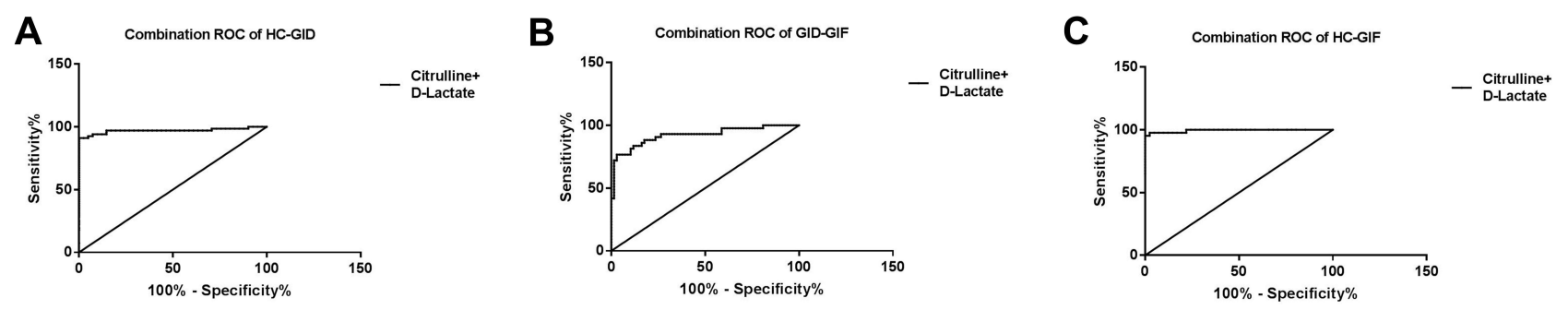

Figure 4 Diagnostic value of serum citrulline combined with serum D-lactate in the diagnosis of gastrointestinal failure (GID) and gastrointestinal failure (GIF). (A-C) ROC curve analysis was used to assess the combination of serum citrulline and serum D-lactate to distinguish GID from healthy control (HC), GIF from GID, or GIF from HC. 
Table 5 Diagnostic Accuracies of Combinations of Serum Citrulline and D-Lactate of Gastrointestinal Dysfunction (GID) and Gastrointestinal Failure (GIF)

\begin{tabular}{|c|c|c|c|c|c|c|}
\hline Groups & Cutoff & Sensitivity (\%) & Specificity (\%) & AUC & $95 \% \mathrm{Cl}$ & $P$ value \\
\hline HC-GID & 0.7049 & 91.04 & 97.56 & 0.9698 & $0.9356-1.004$ & $<0.0001$ \\
\hline GID-GIF & 0.5381 & 76.74 & 92.65 & 0.9210 & $0.8642-0.9778$ & $<0.0001$ \\
\hline HC-GIF & 0.4870 & 95.35 & 97.56 & 0.9943 & $0.9834-1.005$ & $<0.0001$ \\
\hline
\end{tabular}

Abbreviations: $\mathrm{HC}$, healthy control; AUC, area under the ROC curve.

critically ill patients. Besides, there was an association between measured biomarkers (serum citrulline and D-lactate) and subjective clinical assessment of GID/GIF. The combination of citrulline and D-lactate may aid in the diagnosis of GID or GIF to some extent in critically ill patients.

\section{Data Sharing Statement}

The analysis data are available from the corresponding author upon reasonable request.

\section{Ethics Approval and Informed Consent}

This study was approved by the Medical Ethics Committee of Sichuan Academy of Medical Sciences \& Sichuan Provincial People's Hospital, and written informed consent was obtained from all participants. This study was conducted in accordance with the Declaration of Helsinki.

\section{Author Contributions}

All authors made substantial contributions to conception and design, acquisition of data, or analysis and interpretation of data; took part in drafting the article or revising it critically for important intellectual content; gave final approval of the version to be published; and agree to be accountable for all aspects of the work.

\section{Funding}

Research project supported by Science and Technology Department "Clinical significance and application of serum intestinal fatty acid binding protein (I-FABP) combined with serum citrate determination in the diagnosis and treatment of gastrointestinal failure in critically ill patients" (No. 2015SZ0164).

\section{Disclosure}

The authors report no conflicts of interest in this work.

\section{References}

1. Padar M, Starkopf J, Uusvel G, Reintam Blaser A. Gastrointestinal failure affects outcome of intensive care. $J$ Crit Care. 2019;52:103-108. doi:10.1016/j.jcrc.2019.04.001

2. Alukal JJ, Thuluvath PJ. Gastrointestinal failure in critically ill patients with cirrhosis. Am $J$ Gastroenterol. 2019;114 (8):1231-1237. doi:10.14309/ajg.0000000000000226

3. Reintam Blaser A, Jakob SM, Starkopf J. Gastrointestinal failure in the ICU. Curr Opin Crit Care. 2016;22(2):128-141.

4. Sertaridou E, Papaioannou V, Kolios G, Pneumatikos I. Gut failure in critical care: old school versus new school. Ann Gastroenterol. 2015;28(3):309-322.

5. Agarwala R, Rana SS, Sharma R, Kang M, Gorsi U, Gupta R. Gastrointestinal failure is a predictor of poor outcome in patients with acute pancreatitis. Dig Dis Sci. 2020;65(8):2419-2426. doi:10.1007/s10620-019-05952-5

6. Bahri S, Zerrouk N, Aussel C, et al. Citrulline: from metabolism to therapeutic use. Nutrition. 2013;29(3):479-484. doi:10.1016/j. nut.2012.07.002

7. Papadia C, Osowska S, Cynober L, Forbes A. Citrulline in health and disease. Review on human studies. Clin Nutr. 2018;37(6Pt A):1823-1828. doi:10.1016/j.clnu.2017.10.009

8. Crenn P, Messing B, Cynober L. Citrulline as a biomarker of intestinal failure due to enterocyte mass reduction. Clin Nutr. 2008;27 (3):328-339. doi:10.1016/j.clnu.2008.02.005

9. Mifsud F, Czernichow S, Carette C, et al. Behaviour of plasma citrulline after bariatric surgery in the BARIASPERM cohort. Clin Nutr. 2020;12(20):045.

10. Shen LJ, Guan YY, Wu XP, et al. Serum citrulline as a diagnostic marker of sepsis-induced intestinal dysfunction. Clin Res Hepatol Gastroenterol. 2015;39(2):230-236.

11. Fagoni N, Piva S, Marino R, et al. The IN-PANCIA Study: clinical evaluation of gastrointestinal dysfunction and failure, multiple organ failure, and levels of citrulline in critically ill patients. J Intensive Care Med. 2020;35(3):279-283. doi:10.1177/0885066617742594

12. Levitt MD, Levitt DG. Quantitative evaluation of D-lactate pathophysiology: new insights into the mechanisms involved and the many areas in need of further investigation. Clin Exp Gastroenterol. 2020;13:321-337. doi:10.2147/CEG.S260600

13. Kowlgi NG, Chhabra L. D-lactic acidosis: an underrecognized complication of short bowel syndrome. Gastroenterol Res Pract. 2015;476215(10):22.

14. Lei G, Zhang J, Wang X, Chen M. Plasma D-lactate levels in necrotizing enterocolitis in premature infants. Iran $J$ Pediatr. 2016;26(2):e4403.

15. Duzgun AP, Bugdayci G, Sayin B, Ozmen MM, Ozer MV, Coskun F. Serum D-lactate: a useful diagnostic marker for acute appendicitis. Hepatogastroenterology. 2007;54(77):1483-1486.

16. Nielsen C, Kirkegård J, Erlandsen EJ, Lindholt JS, Mortensen FV. D-lactate is a valid biomarker of intestinal ischemia induced by abdominal compartment syndrome. J Surg Res. 2015;194 (2):400-404. doi:10.1016/j.jss.2014.10.057 
17. Shi H, Wu B, Wan J, Liu W, Su B. The role of serum intestinal fatty acid binding protein levels and D-lactate levels in the diagnosis of acute intestinal ischemia. Clin Res Hepatol Gastroenterol. 2015;39 (3):373-378. doi:10.1016/j.clinre.2014.12.005

18. Reintam Blaser A, Malbrain ML, Starkopf J, et al. Gastrointestinal function in intensive care patients: terminology, definitions and management. Recommendations of the ESICM working group on abdominal problems. Intensive Care Med. 2012;38(3):384-394. doi:10.1007/s00134-011-2459-y

19. Godinjak A, Iglica A, Rama A, et al. Predictive value of SAPS II and APACHE II scoring systems for patient outcome in a medical intensive care unit. Acta Med Acad. 2016;45(2):97-103. doi:10.5644/ama2006124.165

20. Raith EP, Udy AA, Bailey M, et al. Prognostic accuracy of the SOFA score, SIRS criteria, and qSOFA score for in-hospital mortality among adults with suspected infection admitted to the intensive care unit. JAMA. 2017;317(3):290-300. doi:10.1001/jama.2016.20328

21. Guo Y, Zhou G, He C, Yang W, He Z, Liu Z. Serum levels of lipopolysaccharide and 1,3- $\beta$-D-glucan refer to the severity in patients with crohn's disease. Mediators Inflamm. 2015;843089(10):28.

22. Li H, Chen Y, Huo F, Wang Y, Zhang D. Association between acute gastrointestinal injury and biomarkers of intestinal barrier function in critically ill patients. BMC Gastroenterol. 2017;17(1):017-0603. doi:10.1186/s12876-017-0603-Z

23. Zhang D, Fu R, Li Y, Li H, Li Y, Li H. Comparison of the clinical characteristics and prognosis of primary versus secondary acute gastrointestinal injury in critically ill patients. J Intensive Care. 2017;5 (1):017-0221. doi:10.1186/s40560-017-0221-4

24. Sun JK, Liu Y, Zou L, et al. Acute gastrointestinal injury in critically ill patients with COVID-19 in Wuhan, China. World J Gastroenterol. 2020;26(39):6087-6097. doi:10.3748/wjg.v26.i39.6087

25. Kartaram S, Mensink M, Teunis M, et al. Plasma citrulline concentration, a marker for intestinal functionality, reflects exercise intensity in healthy young men. Clin Nutr. 2019;38(5):2251-2258. doi:10.1016/j.clnu.2018.09.029
26. Adeva-Andany M, López-Ojén M, Funcasta-Calderón R, et al. Comprehensive review on lactate metabolism in human health. Mitochondrion. 2014;17:76-100.

27. Kuss SK, Best GT, Etheredge CA, et al. Intestinal microbiota promote enteric virus replication and systemic pathogenesis. Science. 2011;334(6053):249-252. doi:10.1126/science.1211057

28. Barzał JA, Szczylik C, Rzepecki P, Jaworska M, Anuszewska E. Plasma citrulline level as a biomarker for cancer therapy-induced small bowel mucosal damage. Acta Biochim Pol. 2014;61 (4):615-631. doi:10.18388/abp.2014_1823

29. Fragkos KC, Forbes A. Citrulline as a marker of intestinal function and absorption in clinical settings: a systematic review and meta-analysis. United European Gastroenterol J. 2018;6 (2):181-191. doi:10.1177/2050640617737632

30. Blaser A, Padar M, Tang J, Dutton J, Forbes A. Citrulline and intestinal fatty acid-binding protein as biomarkers for gastrointestinal dysfunction in the critically ill. Anaesthesiol Intensive Ther. 2019;51 (3):230-239. doi:10.5114/ait.2019.86049

31. Kong C, Li SM, Yang H, et al. Screening and combining serum biomarkers to improve their diagnostic performance in the detection of intestinal barrier dysfunction in patients after major abdominal surgery. Ann Transl Med. 2019;7(16):102. doi:10.21037/atm.2019.07.102

32. Treskes N, Persoon AM, van Zanten ARH. Diagnostic accuracy of novel serological biomarkers to detect acute mesenteric ischemia: a systematic review and meta-analysis. Intern Emerg Med. 2017;12 (6):821-836. doi:10.1007/s11739-017-1668-y

33. Hyšpler R, Tichá A, Kaška M, et al. Markers of perioperative bowel complications in colorectal surgery patients. Dis Markers. 2015;428535(10): 15 .
International Journal of General Medicine

\section{Publish your work in this journal}

The International Journal of General Medicine is an international, peer-reviewed open-access journal that focuses on general and internal medicine, pathogenesis, epidemiology, diagnosis, monitoring and treatment protocols. The journal is characterized by the rapid reporting of reviews, original research and clinical studies

\section{Dovepress}

across all disease areas. The manuscript management system is completely online and includes a very quick and fair peer-review system, which is all easy to use. Visit http://www.dovepress.com/ testimonials.php to read real quotes from published authors. 